\title{
Soziales und politisches Engagement unter schwierigsten Bedingungen
}

\section{L.E. Voigtländer: Armut und Engage- ment. Zur zivilgesellschaftlichen Par- tizipation von Menschen in prekären Lebenslagen. Bielefeld: transcript 2015, 318 S., Print-ISBN 978-3-8376-3135-7, PDF-ISBN 978-3-8376-3135-1, Euro 34,99}

\section{Rezension von Michael Strähle, Wissenschaftsladen, Wien}

Obwohl die Gruppe von Erwerbslosen und Armen recht groß ist, ist ihr Engagement in sozialpolitischen Belangen vergleichsweise gering. Offensichtlich gelingt es Erwerbslosen und Armen nicht, sich als Interessengruppe zu formieren, die anderen Interessengruppen ihre Perspektiven entgegensetzt. Ihr Engagement bleibt zumeist unter dem Radar der Medien. Wenn über Erwerbslose und Arme gesprochen wird, dann als Betroffene, als Subjekte der Fürsorge, nicht als politische Subjekte, als engagierte BürgerInnen. Zwar gibt es Forschungen darüber, weshalb sich relativ wenig Erwerbslose und Arme sozialpolitisch engagieren, doch ist kaum etwas über die Bedingungen bekannt, unter denen dies geschieht. Das vorliegende Buch, eine im Dezember 2013 an der Freien Universität Berlin eingereichte Dissertation, versucht, Bedingungen herauszuarbeiten, die aus der Perspektive der Engagierten fördern oder hemmen.

Anhand von 16 leitfadengestützten Interviews mit Bürgerinnen und Bürgern, die zum Zeitpunkt der Befragung Leistungen der sozialen Mindestsicherung bezogen und sich in unterschiedlichen sozialen Projekten im südlichen Sachsen-Anhalt engagierten, wird untersucht, unter welchen Bedingungen sie sich engagierten, worin ihre Ziele bestanden und inwiefern jüngere Entwicklungen in der Arbeitsmarktpolitik (,Hartz-IV“) sich auf dieses Engagement auswirken. Der Autor des Buches sieht das Problem bürgerschaftlichen Engagements von Erwerbslosen und Armen v. a. durch folgende Fragen umrissen: Das bürgerschaftliche Engagement von Armen ist aufgrund ihrer knappen Ressourcen nie ungefährdet. Diskriminierung zeigt sich auch dort, wo sie aufbegehren. „Worauf lässt sich diese Diskriminierung zurückführen? Was lehrt sie über das Vermögen der (sozialen) Bürgerrechte, Bürger vor ihr zu schützen?" (S. 19) Wie zeigt sich der Sozialstaat gegenüber Anspruchsberechtigten? Werden sie als BürgerInnen oder als Untertanen behandelt? „Inwiefern schlägt sich diese Erfahrung in ihrem Handeln nieder?" (S. 20) Und wie gestaltet sich ,der graue Alltag des Engagements"? (S. 20)

Im Einleitungskapitel werden die Problemstellung, der Forschungsstand, die allgemeine Fragestellung und die Lücke, die das Buch zu schließen beabsichtigt, flüssig lesbar dargelegt. Bürgerschaftliches Engagement wird im Anschluss an Roland Roth definiert und umfasst z. B. Tätigkeiten in Verbänden, Engagement in sozialen Projekten und Initiativen wie Tafeln und Engagement in Selbsthilfeprojekten wie Beratungsstellen.

Der Forschungsstand wurde erhoben in Bezug auf das Ausmaß des Engagements von Bürgerinnen und Bürgern in prekären Lebenslagen und auf die Faktoren, die es ihnen erschweren, kollektiv Interessen zu artikulieren und zu vertreten. Die bekannte soziale Verzerrung bürgerschaftlichen Engagements wird bündig dargelegt. Bürgerschaftliches Engagement ist v. a. für diejenigen attraktiv, die über Ressourcen, ein ausreichendes Einkommen, Bildung und gute gesellschaftliche Beziehungen verfügen. Eine Erwerbstätigkeit macht soziales Engagement wahrscheinlicher, speziell, wenn es nicht stark institutionalisiert ist. Kritischer Konsum ist eher eine Sache von Bürgerinnen und Bürgern mit höherem Einkommen als von solchen in prekärer Lebenslage, die sich dafür unter Umständen nachsagen lassen müssen, sich nicht ausreichend verantwortungsvoll zu verhalten. (Hier hätte der Autor, sofern die Autorinnen und Autoren, auf die er sich bezieht, es nicht bereits getan haben, auch Pierre Bourdieus Analysen heranziehen können, wie sie sich z. B. in den Feinen Unterschieden finden, um dies herauszuarbeiten.) Die ungleichen Bedingungen für soziales und politisches Engagement schlagen sich auch in einer geringeren Wahlbeteiligung sozial Benachteiligter nieder. Auch die politische Partizipation ist ungleich verteilt. Ein weiterer Schwerpunkt der 
Erhebung des Forschungsstandes waren die Probleme der Selbstorganisation und Interessenartikulation Erwerbsloser und Armer. Es gibt keine Erwerbslosenbewegung, nur unterschiedliche, überwiegend lokale Initiativen, die sich darum bemühen, z. B. durch Beratung, einen rasch erfahrbaren konkreten Nutzen für andere Erwerbslose und Arme zu erbringen. Diese Initiativen sind überwiegend nicht in überregionalen Verbänden vernetzt. Die herrschenden Förderbedingungen bevorzugen befristete, projektbezogene Aktivitäten, die sich auf soziale Dienstleistungen konzentrieren. Für überregionale Vernetzungen fehlen überwiegend die Ressourcen. Die Initiativen spielen in sozialpolitischen Auseinandersetzungen kaum eine Rolle, sie kommen in den Medien kaum vor. Aus einem sozialpolitischen Thema (die marginale Rolle von Erwerbslosen und Armen bei der Definition und Diskussion gesellschaftlicher Probleme) wird ein arbeitsmarktpolitisches, wodurch der Spielraum der sozial Engagierten sich erst recht verengt. Ihnen verbleibt es im Wesentlichen, eine sozialrechtliche Auseinandersetzung in eine Durchsetzung von Leistungsansprüchen gegenüber dem Jobcenter zu transformieren. Es wird nicht mit ihnen gesprochen, sondern über sie. Der dritte Schwerpunkt der Erhebung des Forschungstandes galt individuellen und kollektiven Faktoren, auf die sich diese sozialen Verzerrungen zurückführen lassen.

Das Methodenkapitel ist erfreulich ausführlich. Betroffene - zwölf Männer und vier Frauen - wurden als Fachleute ihrer Belange interviewt. Das Forschungsdesign wurde mit potenziellen Interviewpartnerinnen und -partnern diskutiert und gemäß ihren Einwänden und Anregungen adaptiert. Ohne diese Anpassungen wäre ein Feldzugang nicht möglich gewesen; zu groß waren die Befürchtungen, ausgeforscht zu werden und Nachteile zu erleiden. Bei der Stichprobe wurde eine möglichst große Variation angestrebt, um möglichst unterschiedliche Perspektiven zu erhalten. Der Interviewleitfaden findet sich im Methodenteil. Die empirische Analyse wird durch theoretische Diskussionen der Folgen der Hartz-IV-Gesetze auf das selbstbestimmte Handeln Erwerbsloser und Armer ergänzt.
Das Empiriekapitel gilt im ersten Teil den privaten Alltagserfahrungen mit prekärer Beschäftigung und Erwerbslosigkeit, geringem Einkommen und dem damit verbundenen Lebensstandard. Außerdem werden die Erfahrungen mit dem Jobcenter und arbeitsmarktpolitischen Maßnahmen, denen die Interviewpartnerinnen und -partner zugeteilt wurden, dargestellt. Im zweiten Teil wird geschildert, wie diese Mitarbeit in sozialen und sozialpolitischen Projekten aus der Perspektive der Engagierten aussieht: die Anlässe dafür, die Arbeitsteilung, die AdressatInnen, das sozialpolitische Feld und welche Unterstützung sie erhalten bzw. welche ihnen fehlt. Schließlich werden im dritten Teil die typischen Motive von Engagierten sowie Bedingungen benannt, die fördern bzw. hemmen.

Selbst wenn einem manches aus anderen Studien bekannt vorkommt, sind diese leicht lesbaren Schilderungen aufschlussreich, denn sie führen vor Augen, wie es unter prekären Umständen möglich sein kann, ein gewisses $\mathrm{Ma} ß$ an Selbstbestimmung aufrecht zu erhalten, wenngleich nicht immer ohne Widerspruch zwischen den Forderungen, die man erhebt, und den Möglichkeiten, die man sich offen hält, um die eigene Situation wenigstens ein klein wenig zu verbessern. Im Vordergrund des sozialen Engagements steht, anderen Erwerbslosen und Armen zu helfen. Die Motive dafür sind recht unterschiedlich. Im Engagement herrschen Fragen von Einkommen und Auskommen vor. Wer andere Erwerbslose und Arme in Auseinandersetzungen mit dem Jobcenter berät, beispielsweise durch die „Übersetzung“ von Verwaltungsbescheiden, erwirbt Expertise in sozialrechtlichen Fragen und damit auch Handlungsfähigkeit in eigener Sache. Der Kampf um das Nötige gegen eine Bürokratie, von der man abhängig ist und der auch die Mitarbeiterinnen und Mitarbeiter des Jobcenters unterworfen sind, kann verbittern, und die unterschiedlichen Interessen von Betroffenen können zermürben. Keiner der interviewten Erwerbslosen und Armen glaubt, dass sich durch Beschäftigungsmaßnahmen, denen man zugeteilt wird, die beruflichen Chancen verbessern. Auch findet sich vereinzelt Skepsis gegenüber dem eigenen sozialen Engagement, ob es nicht gerade die $\mathrm{Zu}$ stände fördert, gegen die es antritt. 
Sehr lesenswert sind die Kapitel des empirischen Teils, die sich mit dem Umgang mit Menschen aus den Zielgruppen beschäftigen und mit den Motiven sowie förderlichen und hemmenden Bedingungen für ein soziales oder politisches Engagement befassen. Feinfühlig wird darüber berichtet, wie Engagierte sich von denen distanzieren, für die sie sich engagieren. Diese Distanzierungen speisen sich teilweise daraus, dass viele Betroffene nicht bereit sind, sich mit Anderen zu organisieren, um gemeinsam langfristige Verbesserungen zu erreichen. Solche Enttäuschungen im sozialen oder politischen Engagement können dazu führen, sich von Anderen positiv abzusetzen, die sich eventuell nicht in der Lage sehen, (weitere) Rückschläge zu riskieren. Die individuellen Motive für ein soziales oder politisches Engagement werden zu typischen Motiven zusammengefasst. Eine Zusammenfassung fördernder und hemmender Faktoren für Engagement hätte sich nur für jede Person, die interviewt wurde, einzeln angeben lassen; zu unterschiedlich sind die Situationen, Ansprüche und Probleme dieser Personen. Wohl aber ließen sich fördernde und hemmende Bedingungen herausarbeiten und detailliert beschreiben: verfügbare materielle und immaterielle Ressourcen, Gestaltungsspielräume, der Druck auf Erwerbstätige und Erwerbslose, die Hilfsbedürftigkeit der Adressatinnen und Adressaten, die Art der Verrechtlichung und die Bürokratisierung des Konfliktes um das Existenzminimum sowie die Resonanz, die Erwerbslose und Arme in Gesellschaft und Öffentlichkeit erfahren.

Eine Literaturstudie zu den sozialen Rechten Erwerbsloser und Armer und wie sie sich auf deren soziales oder politisches Engagement auswirken, ergänzt die empirischen Erhebungen. Voigtländer arbeitet heraus, dass soziale Rechte als Bürgerrechte zu verstehen sind, die Menschen in Notlagen davor schützen sollen, der Entscheidungsgewalt von Behörden ausgeliefert zu sein und nicht selbstbestimmt handeln zu können. Er legt dar, wie sich die Änderungen in der Arbeitsmarktpolitik durch die Hartz-IV-Gesetze negativ auf die sozialen Rechte und auch auf die Arbeitsbedingungen der Sachbearbeiterinnen und Sachbearbeiter im Jobcenter niederschlagen: Die Anspruchsberechtigten werden bevormundet, ihre Lage hat sich verschärft. Entsprechend scharf werden die negativen Konsequenzen dieser Entwicklung auf die bürgerschaftliche Teilhabe festgehalten. Armen fehlt es an Ressourcen für soziales Engagement, ihr gesellschaftliches Ansehen ist gering. Mildtätige Hilfe kompensiert den Abbau sozialer Rechte.

Im Abschlusskapitel werden die gewonnenen Erkenntnisse in Form von nachvollziehbaren Thesen zusammengeführt. Dem Autor gelingt es, durch erhellende Einblicke in die Erfahrungen sozial und politisch engagierter Menschen in prekären Lagen Sachverhalte, die auf abstrakter Ebene vertraut sind, in einer respektvollen Weise zu veranschaulichen, die zum Nachdenken über die Lage von Erwerbslosen und Armen einlädt, ja, zwingt. Jedoch wünscht man sich angesichts der skizzierten Spannungsfelder, in denen sich das soziale und politische Engagement der Interviewten bewegt, dass Voigtländer benannt hätte, wo, abgesehen von Gesetzesänderungen, angesetzt werden könnte, um förderlichere Bedingungen für das soziale und politische Engagement Erwerbsloser und Armer zu schaffen. Dieses kleine Desiderat mindert den Wert dieser Studie keineswegs. Sie ist nicht nur für hauptberufliche in der sozialen Arbeit oder in der Arbeitsmarktbzw. Sozialpolitik Tätige empfehlenswert, sie bietet auch wertvolle Anregungen für alle, die sich bemühen, Beteiligungsmaßnahmen möglichst inklusiv zu gestalten, folglich auch für die partizipative Technikfolgenabschätzung. 